Educational Research for Social Change (ERSC)

Volume: 5 No. 2, September 2016

pp. 151-164

ersc.nmmu.ac.za

ISSN: 2221-4070

\title{
Sustainability of International Collaborative Partnerships in Education: A Personal Research Journey
}

\author{
Petra Engelbrecht \\ Faculty of Education Sciences, North-West University \\ petra.engelbrecht50@gmail.com
}

\begin{abstract}
The growth of international research and development partnerships in education has raised significant challenges regarding the conceptualisation, dissemination, and sustainability of international projects that transcend international borders. This article focuses on an analytical autoethnographical account of collaboration in an international project on improving the quality and relevance of the teaching practice component of initial teacher education programmes in Palestine. Collaborative partnerships are conceptualised as the synergy created when the perspectives, resources, and skills of a group of people are combined. Analytical autoethnography is used as the methodological approach, in which personal narrative and research are combined in a variety of ways, for example, extending initial analysis of the dynamics of collaborative partnerships by combining it with own experiences as well as with personal reflections taken one year after the project came to a close. Partners' characteristics and the relationships between partners, overall partnership characteristics, and the role of the complex geopolitical environment, with an emphasis on the complexity in the development of culturally competent work, were identified as themes. The conclusion reached is that synergy for sustainable international partnerships is influenced by cultural competence and positive interpersonal connections, within which the relevant power differentials are recognised and cultural assumptions are made.
\end{abstract}

Keywords: Collaboration, collaborative partnerships, sustainability, autoethnography, international development programmes, cultural competence

Copyright: @ 2016 Engelbrecht

This is an open access article distributed under the terms of the Creative Commons Attribution Non-Commercial License, which permits unrestricted non-commercial use, distribution, and reproduction in any medium, provided the original author and source are credited.

\section{Please reference as:}

Engelbrecht, P. (2016). Sustainability of International Collaborative Partnerships in Education: A Personal Research Journey. Educational Research for Social Change, 5(2), 151-164. http://dx.doi.org/10.17159/2221-4070/2016/v5i2a10 


\section{Introduction}

The growth of international collaborative development and research programmes owes much to a greater focus on transcultural research, funding in this regard, and the concern of universities to extend their international visibility (Rigby \& Edler, 2005). It has also raised significant challenges and concerns regarding the conceptualisation and dissemination of international educational research, its sustainability, and the development of collaborative partnerships that transcend international borders. Furthermore, as those of us who have been closely involved in the development of international educational research and development projects have learned, developing sustainable international partnerships has been difficult, and has often been fraught with issues. These issues are not only related to processes of funding, knowledge production, and who the ultimate beneficiaries of the research will be, but also to partnership relationships as well as transcultural communication and intercultural learning.

In this article, I focus on revisiting my field notes and personal reflections from a reflexive diary (ethnographic account), that I kept during an international project in education in Palestine that came to an end in 2014, in order to explore aspects that could possibly have influenced the development of sustainable international partnerships in education that brought about change. The purpose is to analyse my own reflections during the project and after it came to an end and, by connecting the personal to the cultural in this way, possibly contribute to further development of knowledge in this field.

The project in Palestine cannot be characterised as a research project as such, but rather as an externally funded knowledge exchange project in education, with a strong emphasis on evaluation research in order to monitor progress and evaluate the impact of the project. As this project came to a close, it generated new projects and influenced the development of revised policies on teacher education. The dynamic relationship between international collaborative projects in higher education and the nature, scale, sustainability, and quality of our collaboration in bringing about educational change in this project has also become clearer. I, therefore, increasingly realised that the role that quality of collaboration played within this project, including the willingness of individuals and collective team groups to transcend international sociocultural boundaries, cannot be underestimated. The challenging path that this partnership followed enabled the greater international project team, as well as the Palestinian project team, to accomplish much more than we would have been able to achieve as individual researchers or as consultants at individual universities. Furthermore, my personal belief in the integral connection between the quality of human relationships in complex international educational research settings and the validity of processes in projects aimed at bringing about social change was confirmed.

\section{Brief Description of the International Project in Education}

The main objective of this knowledge exchange project was to improve the quality and relevance of the teaching practice component of teacher education programmes for primary school-aged pupils in Grades 1-4 in Palestine (West Bank and Gaza). In pursuance of this objective, the World Bank funded a Teacher Education Improvement Project (TEIP) in 2010, for which an international cross-faculty, cross-university team successfully bid to act as international consultants. Notification of the contract was received in March 2011, and the project came to a close in 2014. Strategic planning to achieve the overall goal of this project within a period of about 36 months was informed by a coherent body of knowledge derived from international research and practice in teacher education, whole-school improvement, and continuing professional development. The approach of the international consultants in the period 2011 to 2014 in assisting, supporting, and advising the four participating universities in the West Bank and Gaza, as well as colleagues in the Ministry of Education and Higher Education in Ramallah, was from the outset framed within a transparent and participatory, 
collaborative partnership approach, based on recognition of the equality of all partners and development of respectful professional relationships (Engelbrecht, 2007; see Mertens, 2009). The complex geopolitical demands of the project led to the decision to use a mixed-methods research design within a pragmatist research paradigm to monitor progress and to, ultimately, evaluate the impact of the intervention. Due to the more complex, multicomponent nature of the project, there was a need to achieve an in-depth understanding of how our Palestinian partners interpreted their experiences in the project and, specifically, their context-specific perspectives and interpretations of progress during the project (see Merriam, 2009). A conscious decision was therefore taken to place greater emphasis on qualitative data collection methods, with the result that qualitative data had a greater weighting in all stages of evaluation of the impact of the intervention (Engelbrecht, Wilson, \& Mahon, 2014).

The international consultancy team consisted of four members. Each member in the team had a specific area of expertise relevant to the development of the project, and outcomes and roles in the project were, therefore, formulated accordingly: expertise in mentoring, teaching practicum, and pedagogy, as well as international expertise in teacher education programmes in higher education, and monitoring and evaluation research in complex community contexts, and one of the members could speak Arabic. In the West Bank and Gaza, our project partners consisted of academics at four universities, Grades 1-4 classroom teachers in 50 schools, and the Palestinian Department of Education in Ramallah under the indirect leadership of the Minister of Education. We collaborated closely both with a small group in the Ministry of Education and with the academics at the four universities who were all regarded as having expertise in initial teacher education programmes, while collaboration with the Grades 1-4 classroom teachers was less intense and was mediated mainly by the academics at the universities concerned.

\section{Conceptual Framework for International Research Collaboration and Partnerships}

As mentioned earlier, the growth of collaborative research owes much to the provision of funding from governments and international aid organisations, as well as the recognition within universities of the potential of international collaborative partnerships that enable partners in different countries to support each other by combining and capitalising on their complementary strengths and capabilities.

Collaboration has generally been defined as a process that enables individuals and organisations to combine their human and material resources in order to accomplish outcomes that they are unable to achieve on their own. However, generalisations about collaborative research are not easy due to considerable complexities and variety in approaches in diverse contexts. Collaborative research can nevertheless be defined as building multisectoral networks that link researchers with complementary assets and knowledge across institutions and countries with the express purpose of utilising research as a strategy for collaborative problem solving in a specific field of study (Beaver, 2004; Chan, 2002; Rigby \& Edler, 2005). Research partnerships have been an increasingly dominant concept in the literature, signifying the attainment of greater equality in collaborative team efforts in development and research projects and the way this can enhance sustainability (Glasbergen, Biermann, \& Mol, 2007). Partnerships within research collaboration can therefore be represented as a core principle or an attitudinal stance based on recognition of the equality of all partners - not only in the project team, but also in ongoing relationships in the communities in which these projects take place (Engelbrecht, 2007; Mertens, 2009).

However, structuring the discussion on the nature, significance, and internal dynamics of collaboration within either a development or a research project calls for a conceptual framework within which to comment on interaction and project processes because, without such a framework, this article would 
simply take the form of a simple description of the sequence of events without identifying their relevance (Jeffrey, 2003). Although there is a variety of approaches to conceptualise the functioning of research partnerships (e.g., Hodgson \& Standish, 2006; Jeffrey, 2003; Rigby \& Edler, 2005), Lasker, Weiss, and Miller (2001) emphasised that there is a need to conceptualise and evaluate the optimal outcome of partnership functioning that captures the mechanism that makes research collaboration especially effective. They identified synergy (the power to combine the perspectives, resources, and skills of a group of people) as the optimal outcome of partnership functioning that gives collaboration its unique advantage. The synergy that a collaborative partnership achieves, according to Lasker et al. (2001), is reflected in the way partners think about the partnership's goals, strategic planning and evaluation, the types of actions carried out, and particularly the relationship between the partnership and the broader community. Determinants of partnership synergy include resources, partners' characteristics (including relationships between partners), specific partnership characteristics, and the external environment.

For the purposes of this article, sustainable collaboration will be discussed within this conceptual framework, which also guided the analysis and interpretation of findings. With reference to this project and the relevant determinants of partnership synergy, resources should include available basic resources (e.g., financial and in kind resources such as computers and books) and, beyond these basic resources, a broad array of skills and expertise are needed to engage partners, support the collaboration process, and manage the project as a whole. In order to sustain the development of this specific project and the envisaged collaboration, adequate funding was provided by a World Bank grant, and strategic thinking about the implementation of the project focused on bringing a broad array of skills and expertise together-not only with reference to the focus of the project, but also to include colleagues with experience in community relationships and communication, information technology, knowledge of Arabic and the cultural and political history of Palestine. Because partners are the source of most partnership characteristics (their skills with reference, for example, to connections and credibility), we focused on actions related to the identification, recruitment, and active engagement of partners-not only within the international consultancy team but also within the wider project team - with a specific range of perspectives in the overall project that could stimulate and develop responsive ways of thinking about initial teacher education programmes in complex settings, and who could collaboratively develop and implement comprehensive actions in this regard. The development of meaningful relationships therefore played a major role not only during the planning stage of the project but also in the subsequent phases of the project (Engelbrecht et al., 2014; Lasker et al., 2001).

\section{My Personal Research Journey Research design and methodology}

In this article, I use analytical autoethnography as the methodological approach because it allows me to blend personal narrative and research in a variety of ways, for example, extending my initial analysis of the dynamics of collaborative partnerships by combining it with my own experiences as well as my reflections after the project came to a close. My research design is therefore exploratory in nature and should be treated as a work in progress, forming the development of a means of further knowing and the discovery of examining self-other relationships (see Richards, 2012; Tomaselli, 2015).

According to Anderson (2006), key features of analytical autoethnography include the following:

- The researcher is a complete member in the social world under study,

- Sustained analytical reflexivity that involves an awareness of reciprocal influence between the ethnographer and the setting and informants is used, 
- The researcher is visible in the text of the group she or he is studying,

- There is dialogue and engagement with informants beyond the self and, finally,

- There is a commitment to an analytical analysis.

However, it needs to be noted that in this project, the researcher played a dual role as a group member: I played the role of project leader and was, therefore, placed in a position of power and privilege. Hence, the necessity of recognising myself in the process and being continually and consciously aware of relevant diversity divides in conversations and behaviours while, at the same time, engaging in these conversations and behaviours (see Mertens, 2009; Tomaselli, 2015). By choosing this qualitative methodology, I chose to make explicit use of my own positionality, involvement, and experiences as an integral part of the project in an effort not only to build critical self-reflexivity, but also to enable me to develop a lived recognition of the research process and all research partners. It is therefore important to note that within the more analytical autoethnographical design of this article, assumptions about reality (ontology) are that multiple realities are shaped by social, political, cultural, economic, gender, ability/disability and other values, and that knowledge (epistemology) is socially and historically constructed and situated (see Mertens, 2009).

\section{Description of myself as a researcher}

Acknowledgement that there is no value free interaction between human beings, and that knowledge of the self is a requirement for doing any type of valid research and evaluation work, is necessary. The importance of this "self-in-dynamic-context" learning and development journey, as described by Symonette (2004), became clear to me during this research journey and previous research journeys, and I regard the journey as a continuing one. The result is that the deepening awareness and knowledge as "self-as-instrument" and "lifelong learning project" has become an integral part of me as a researcher-and as a person. Furthermore, as a South African, my own research approach in the past 25 years has developed during the country's times of conflict and social change (binary opposites of self and other) as well as in recent times, globally, in developing, as well as so-called established, democracies (see Mertens, 2009).

Being the only South African in the international project team also made me aware of the fact that cultural realities and interpretations of events, not only within the umbrella collaborative team (colleagues from Palestine as well as the United Kingdom) but also within the United Kingdom/South African group, might be variable, changing, and in some instances even contradictory. As a result, I was, from the outset, aware at a deeper reflective level of the role of reciprocal influence between myself as ethnographer, and my setting, and project partners, including both United Kingdom and Palestinian partners, and the need for sustained self-conscious introspection in order to understand both myself and my colleagues better (see Anderson, 2006).

\section{Ethical considerations}

Formal approval for the research was provided by the donor organisation, the Palestinian government, relevant universities in Palestine, and the relevant university in the United Kingdom where the project was registered. Taking note of recent debates on researching ethically across cultures (e.g., RobinsonPant \& Singal, 2013), collaborative reflexive analysis during discussions within the international consultancy team as well as with project partners continuously highlighted the need to recognise and react to issues of power and possible unequal and unethically positioning of all participants (as discussed in the findings). 


\section{Major data sources}

Primary sources of data included my extensive field notes as well as a personal reflexive diary, which I kept during the project and which after completion of the project, enabled me to develop a deeper understanding of my own actions and perceptions as well as those of my colleagues in the project. Field notes included, for example, detailed notes of project meetings (including discussions that focused on reflections of our own interpretations as "outsiders" of our actions in the field), which were held at the end of every day during field visits.

Other data collection methods used, for the purposes of this article, included the following:

- A literature review about collaboration in research and the development of international research partnerships, and

- Minutes of meetings held over a period of five years.

\section{Data analysis}

As mentioned earlier, a characteristic of analytical autoethnography is not simply to document personal experience, but to use empirical data to gain insight into broader sets of social phenomena and, in this instance, into sustainable international collaborative partnerships. Essentially using an inductive qualitative content analysis after completion of the project, I searched for insights not only into emerging themes specifically based on the conceptual framework for partnership dynamics adopted for this study (see Lasker et al., 2001), but also into what other themes emerged throughout the analysis (see Merriam, 2009) with the assistance of analytical autoethnography.

The key features of analytical autoethnography assisted in the data analysis in the following ways (Anderson, 2006). Being a complete member researcher played a major role in the data analysis because the necessity of mentally and physically documenting my own activities could divert my attention at times from the other participants, and I needed to be aware of this challenge. Furthermore, my own understandings, both as a member and as a researcher, needed to emerge not from detached discovery but from engaged dialogue that reflected my own awareness of reciprocal influence between myself, project settings, and perceptions. My reflexive notes clearly indicate at times the stress involved in self-conscious introspection, and the need to develop a better understanding both of myself and all my partners. As a result, the findings tend to reveal myself as an active researcher in the text who grapples at times with relevant issues related to collaborative membership and participation in the project. I therefore had to remind myself of the potential for selfabsorption and to not lose sight of the importance of dialogue with others. With specific reference to the overall focus of this article (synergy in the development of sustainable collaborative partnerships), the defining characteristic of the data analysis has been to gain insight into a broader set of data transcending practices-using multiple data collection strategies directed towards refinement and extension of our existing knowledge of the development of international collaborative research partnerships.

\section{Trustworthiness}

Using my personal journals and field notes as the primary instruments for data collection and analysis enabled me to expand my understanding of the process of the project, with a specific emphasis on the transcending of international research borders, as well as developing research partnerships. However, being aware that my own biases, or subjectivities, can have an impact on the trustworthiness of my analysis, I have used member checks (done by members of the research project team) as well as peer 
review as strategies to check the congruence between emerging findings and tentative interpretations (see Merriam, 2009).

\section{Findings}

Using Lasker et al.'s (2001) conceptual framework, the following themes were identified: partners' characteristics and the relationships between partners; overall partnership characteristics; and the role of the external environment, with a strong emphasis on the complexity in the development of culturally competent work. The key features of analytical autoethnography (being a member researcher, using analytical reflexivity, narrative visibility of myself, dialogue with informants other than the self, and commitment to theoretical analysis) furthermore assisted in the analysis and findings.

\section{Partner characteristics and the relationships between partners}

Lasker et al., (2001) pointed out that partners' characteristics and relationships influence professional interpersonal relationships in a complex way, and that heterogeneity and level of involvement of partners are important. Heterogeneity, in terms of both gender and culture, played a major role in the development of relationships between participants in this project. The international project team consisted of three British citizens (two women and one man) and one female South African (who was also the project leader). Our Palestinian partners were all Arabic-speaking, and were working in the Ministry of Education in Ramallah, four universities in the West Bank and Gaza (academics and student teachers), and as teachers (mostly female) in Grades 1-4 classrooms (50 schools). Within and between these groups were various levels of involvement and connections to political decision makers, government agencies, and the external funder of the project, with some partners having the influence and the ability to bring people together for meetings and other project activities. Both minutes of meetings, observations described in field notes during initial workshops, as well as my reflexive diary indicated that, initially, the different groups in Palestine became polarised into two main factions - the group in the Ministry of Education, and the group of academics-with an underlying feeling of scepticism, bordering at times on suspicion, from the group of academics in particular, regarding the development and sustainable implementation of an alternative approach to the traditional approach in teacher practicum in initial teacher education programmes. Because these dynamics had serious implications for the development of strong working relationships among partners and the resultant achievement of high levels of synergy and the successful completion of the project, developing relationships of trust and negotiating power structures was a complex process that involved multiple nuanced layers of power (see Robinson-Pant \& Singal, 2013). My analysis of my field notes and my reflexive diary indicates that learning to negotiate these power hierarchies within the relevant structures as well as stimulating and supporting new locally relevant responsive ways of thinking about solutions, and copartnering implementation of new and comprehensive actions, initially proved to be difficult. My personal reflective notes as well as minutes of meetings and extensive field notes indicate that the two risks listed below were identified.

\section{Language as a critical issue}

With regard to the issue of language, the following is a quotation that I recorded in my reflexive diary:

As evaluators we must realise that there is much more to how a language functions in a culture and that the mere translation of certain concepts or measures will not fully capture the experience of the participants [...] if an evaluator is not fully aware of a particular culture and how their linguistic patterns shape the behavioural patterns of the individual from that culture, then the evaluator cannot make logical assessments about the impact of a certain intervention. (Guzman, as cited in Mertens, 2009, pp. 238-239) 
A major challenge to the development of partnerships was that the language of the evaluators (English) was different from the language of the respondents (Arabic), and it was necessary to rely heavily on collaboration with Arabic-speaking colleagues. Because many of the participants in the project did not speak English, open-ended questions were translated and most of the interviews were conducted in Arabic. Two Arabic-speaking colleagues in the United Kingdom played an integral role in translating interviews into English.

As a result, as the main data analyst in the evaluation and also an English second language speaker, I needed to be careful in my interpretation of the data and specific concepts, and I needed to acknowledge that my interpretation should be based on a thorough understanding of the culture that informed production of the data. It became, for example, increasingly clear to all of us that there are a number of cultural and linguistic ambiguities and differences between Arabic and English with regard to concepts and terms. An example that illustrates this well is the term mentor. As Kanan and Baker (2002) pointed out, there is no exact Arabic equivalent for the term mentor (Field notes: "What is this thing called mentoring, I do not understand it all!" [Participant from a university in the West Bank, initial workshop on mentoring in teacher education programmes in Ramallah]). As a result, minutes of meetings and field notes of initial discussions on the important role that mentors, and the development of mentoring skills, played in this project focused more on the characteristics and roles of mentors within the project so as to develop a shared understanding of the term that fitted within the existing cultural and social mores within the community. The development of a collective and comprehensive set of reference terms therefore formed an integral part of the project process.

\section{Cultural competence and sensitivity}

A quotation that I referred to frequently in my reflexive diary, especially on days during field trips that were particularly challenging, was the following: "Cultural competence is not a static state. It is a journey in which the researcher/evaluator develops increased understanding through self-reflection and interaction with members of the community, of the reality of differential access to power and privilege" (Mertens, 2009, p. 90).

Regarding our understanding of the cultures involved (including the differences in home language), programme facilitators and project participants needed to demonstrate cultural sophistication in all aspects of their work in the project. In order to illustrate multicultural validity-not only in the codevelopment of an effective teaching practicum framework for the Palestinian context but also in data collection for progress and impact evaluation, analysis of the data, and discussion of the resultsmembers of the international project team therefore increasingly focused on the development of positive interpersonal connections including trustworthy communication with reference to language differences (as mentioned earlier) as well as conceptualisation and facilitation of culturally congruent change as far as possible in the development of teacher education practices. My extensive reflexive diary indicates, for example, that the international consultancy team shared our own confusion regarding issues related to what could be regarded as culturally congruent change with one another, and acknowledged the need to find support in developing our own understanding in an ethical manner. My diary has the following comment after an initial project meeting:

I need to meet as a matter of urgency with my colleague JS (a Palestinian colleague in the United Kingdom and not connected to the project at all) to discuss our first impressions of what we perceive as our own lack of in-depth understanding of what could possibly be regarded as culturally congruent change and power differentials in the Palestinian context. 
We therefore continuously endeavoured to make appropriate cultural assumptions, in collaboration with our Palestinian colleagues, in the design and implementation of the initial teacher education programme, as well as the monitoring of progress in its implementation.

Cultural competence and sensitivity also form an essential part of collaborative partnerships that are characterised by trust, respect, communication, and a shared vision, and my field notes and journals clearly indicate that at times all the participants struggled to achieve trust and transparency. Not only cultural differences, but also personality differences, contributed to this process and understanding the power differentials in Palestinian society, with specific reference to formal structures within the education system and gender, also created challenges at times. It was vital that the researchers knew themselves; with specific reference to myself, knowing myself in relation to my colleagues and partners, and my increasing self-awareness of my own willingness (and reluctance at times) to acknowledge the potential of miscommunication, as well as imbalances of power and privilege related to culture, cannot be emphasised enough. I therefore had to develop the analytical strategies to recognise and acknowledge, as well as change, long-standing habits-which before then, I was unaware could be regarded as challenging. For example, taking notes during meetings was initially regarded by some participants as an invasion of their privacy, and after becoming aware of this challenge and using dialogue with other participants as a way to address this challenge, I had to acknowledge the differing assumptions of colleagues that privacy in interviews and discussions is not only associated with physical space but, particularly within the unique geopolitical setting of this project, with a sense of security. I also increasingly realised that, especially at stressful moments during workshops and general discussions, I tend to focus more on process rather than on emotions. Interestingly, I also unconsciously found emotional release by at times changing the language I was writing my field notes in, from English to Afrikaans (my home language), and in this way I voiced my own stress during stressful discussions. Becoming aware of the fact that I wrote in Afrikaans during stressful moments enabled me over time to immediately recognise conflicting values and beliefs between individual partners, and to find a balance between reflection and action when needed so as to manage chaos and maintain order. Instances when such balance was required were, for example, during heated exchanges in Arabic between Palestinian colleagues at workshops or in meetings. The role that my United Kingdom project partners played-particularly their willingness to collaborate with me in gaining the trust of our Palestinian colleagues, self-reflect during our discussion sessions at the end of every day during our site visits, and openly discuss our own personal challenges, particularly regarding our ability to be flexible in strategic planning issues-enabled us to build rapport between the four of us. This rapport, based on the willingness to critically self-reflect, cultural competence, and sensitivity, contributed to partnership synergy and the sustainability of the project (e.g., the new teacher education curriculum will now be implemented in all Palestinian universities, and we were invited to do a follow-up project). It also contributed to our ability to synthesise creative ideas, and to deal with personal and professional differences in various project settings in a constructive manner, without exercising premature closure for the purpose of maintaining peace. In this way, we could demonstrate our sensitivity for different values and expectations.

\section{Partnership characteristics}

Certain attributes of the partnership as a whole are, according to Lasker et al. (2001), likely to have a strong influence on the development of partnership synergy. The following attributes of the partnership at micro, mesa, and macro levels within this project as a whole, had a strong influence on the level of partnership synergy: leadership, including leadership styles at all system levels; administration of the project process at project level; management of deadlines; and overall governance were particularly relevant in this regard. One of the key challenges was the modelling of my own leadership style, which could be defined as more participatory, in contrast to the more authoritarian leadership style at various partnership levels in the context of our project implementation. This more participatory leadership style of mine was initially regarded by some of our 
Palestinian partners as not assertive enough. Furthermore, there was an expectation that the international team would provide the "answers" and materials, which is characteristic of countries that rely heavily on donor funding; the result is that research in this area indicates that ownership of the process, as well as sustainability of interventions, is regarded as negligible (see Srivastava, De Boer, \& Pijl, 2015). An analysis of minutes of meetings, field notes, and my reflexive diary therefore indicate that development of a participatory partnership approach was initially challenging and that, as a result, we had to extend our participation by a further year. An example from the minutes of a meeting with Government officials in Ramallah: "It was pointed out by $M$ (a high ranking official) that the international consultancy team is in general regarded as knowing more about teacher education and therefore should provide very strong guidance and leadership at all times." Developing collaborative partnerships based on sustainable equitable relationships in which sociocultural and power differences were acknowledged demanded participation and a shared vision at every stage of the project (see Heron \& Reason, 2006). Dedicated and focused working sessions during which all participants were encouraged to articulate their reservations, collectively search for solutions to challenges, and develop a shared vision-as well as respect for the limitations of own and others' understandings-played a critical role (see Jeffrey, 2003). Development in this respect was demonstrated after 18 months when the answer to the initial question, of what the expected outcomes of the project for Palestinian education should be, changed from an emphasis by Palestinian colleagues on "you teach us what we should do" to "we as a group are developing a new approach to initial teacher education practicum in the Palestinian context."

\section{External factors}

As mentioned in the discussion of the conceptual framework for this project, the ability of a partnership to flourish can be influenced not only by internal challenges as discussed above, but also by factors in the external environment that are beyond the ability of the partnership to control (Lasker et al., 2001). The complex geopolitical context (the West Bank and Gaza) within which this study took place, frequently complicated implementation of the project, and had an impact on progress as well as on the emotional and physical well-being of all team members in the project. A comment in field notes during a face-to-face meeting with partners in Ramallah:

We all look stressed and some of our team members come across as really tired but it is positive to note that all express their enthusiasm for the project despite difficulties today in travelling to project meeting due to security constraints at the moment within these parts of the West Bank.

One of the most challenging contextual issues that the project team members in Gaza and the international project team therefore had to contend with was the fractured political geography of Palestine, as well as the security implications of the Occupation. Restrictions on mobility periodically influenced progress in the project. For instance, the international project team did not receive permission from the Israeli authorities to enter Gaza during some of their field and site visits, and partners from Gaza were, at all times, not allowed to travel to the West Bank. Participation in workshops and discussions as well as interviews was, therefore, at times limited to videoconferencing or Skype, which was frequently affected by poor connections or electricity blackouts. Comment in reflexive diary:

We are all totally exhausted tonight, both mentally and physically, after a long day trying to discuss project development with colleagues in Gaza via video links; there were at least three interruptions due to power failures on the Gaza side. 
As a result, we had to arrange for one face-to-face meeting with the project team in Gaza to take place in Cairo. We also arranged for two visits by members of the global Palestinian project team to visit the United Kingdom for collaborative workshops and school visits. We were never sure how many of our colleagues would actually attend these meetings in the United Kingdom because of the way visas for Palestinians were granted. My notes continually emphasised that I as an individual, as well as a group member and the project leader, had to take care not to get involved in complex political issues, and that our point of departure as international consultants was a focus on the development of quality education for all.

\section{Discussion}

A collaborative partnership's level of synergy is reflected in the extent to which energy is maximised and every partner is engaged in and committed to the vision of the project they are involved in (Lasker et al., 2001). As highlighted by Mertens (2009), cultural competence and resultant trusting professional relationships in the development of international partnerships across cultures are critical dispositions, which are related not only to an individual partner's ability to accurately represent reality in culturally complex communities, but also to a commitment to collaborative partnership synergy. My analysis of the data including my own reflective notes made after completion of the project, clearly indicate that over time the notion of a core group of British, South African, and Palestinian partners, journeying together in a spirit of reciprocity with a shared vision regarding quality education, affected the accountability of both the international project team and our Palestinian colleagues in terms of the outcomes in this project. The most salient benefit of the development of cultural competence therefore was the growth of effective, trusting, professional collaborative relationships, and a culturally responsive approach to evaluation of the project, which was perceived as legitimate by the community involved in the project. Despite the fact that other systemic factors that influence the development of sustainable collaborative international partnerships (for example, complex external geopolitical issues) did figure in our project, responsive partner and partnership relationships-as factors contributing to the dynamics of these culturally responsive partnerships-provided the integral connection between the quality of these relationships and the sustainability of project results in order to bring about change in the teacher practicum in initial teacher education programmes.

The following played a major role. Mertens (2009) pointed out that people need to feel that they are receiving as much valuable energy as they are giving. Determining reciprocity in international education project partnerships is therefore a complex matter because partners' ideas about equity and fair exchange usually involve diverse beliefs about what is right and what is wrong - and they are influenced by trust. Interpersonal (transactional) trust components, such as trust of character (contractual trust), competence trust, and communication trust (trust of disclosure and maintaining confidentiality) form an integral part in the development of reciprocal participatory and collaborative development and research partnerships. It is therefore critical that one be aware of the consequences of making promises that one possibly cannot keep, as well as of systemic power differentials involved. A transparent collaborative partner and partnership relationship ethic forms the core and ethical issues regarding, for example, who decides the agenda, who has the power in the partnership relationship, how the partnerships will be formed, how they can work fairly, and who owns the data need to be discussed from the outset. The need for genuine respect for complex contexts, practices, and paradigms of thinking, as an integral part of cultural competence in the development of willingness among all the partners to engage in and build the partnership, cannot be overemphasised (Mertens, 2009).

As my reflective journey after completion of the project indicates, searching for common ground in the development of culturally competent partnerships in order to transcend international research borders is challenging and, as I hope was illustrated earlier, it involves acknowledgement of the 
complexity of the community within which one is working. Tensions and the potential for conflict arise when the process of taking ownership of a project like the one discussed in this article proves to be a challenge. As is illustrated in the findings, giving explicit attention to valuing differences, and effective and purposeful conflict resolution and mediation are therefore important strategies in the development of successful collaborative partnerships where ownership is taken by the communities involved (Heron \& Reason, 2006; Mertens, 2009).

Furthermore, an important element in transcending international research and development borders is the ability of all partners to be critically self-reflexive, and the ability to develop high quality awareness to understand themselves and others. Grappling, for example, with my own notions of power differentials in a geopolitically complex project context, and how they relate to authentic membership in the umbrella project team and equitable participation, contributed to the process of understanding and knowing myself as a researcher and an evaluator. Questions I reflected upon using self-conscious introspection and analytic reflexivity guided by the desire to better understand myself and others included the following: my own personal beliefs and assumptions about what, for example, is reality (ontology); what my own as well as my colleagues' assumptions about the nature of knowledge are (epistemology); how I as well as my colleagues understand the dynamics of power differentials when authentic collaborative research is undertaken; whose voices are raised, and whose voices are or should be heard; and how we as project partners can mediate these voices as issues of representation in complex general and research project contexts (see Anderson, 2006; Mertens, 2009). As mentioned in the findings, knowing oneself in relation to the community you are involved in also implies that uncritical subjectivity can be a barrier to transcending research borders. Strategies to address subjectivity include, for example, the development of collaboration strategies that are based on equal partnerships that allow every voice to be heard, managing diversity in a respectful way, and finding the balance between reflection, participation, and action-as well as between chaos and order. Withdrawing from an international research project because of one's own concerns about, for example, the research process, cultural differences, or ethics would therefore be ethically preferable to staying in a project where one feels that one's own beliefs will be compromised (Robinson-Pant \& Singal, 2013).

\section{Limitations}

The evidence upon which my conclusions concerning the sustainability of transcending international educational project borders, and the role that collaboration between researchers plays in this regard, is derived mainly from an emerging analytical autoethnographical description of my own experiences as expressed in field notes, reflexive personal journals, analysis of minutes of meetings, and impressions of the interactions between the partners involved (Jeffrey, 2003). As a result, my conclusions should be regarded with some discretion, for the following reasons:

- Firstly, my analysis involved limited systematic data collection in an empirical sense;

- Secondly, there has been no formal attempt at this stage to analyse the psychosocial aspects of the collaboration in more depth; and

- Thirdly, the pressure of purposefully documenting and analysing action, as well as purposefully engaging in it, could have diverted my attention from what Anderson (2006) called the embodied phenomenological experience.

\section{Final Reflections}

I fully acknowledge the complexities involved in transcending international research borders in education, and the limitations in my discussion of these complexities. However, against the 
background of an analytical autoethnographical research design in which an analysis of my own understandings, both as a member and as a researcher of complex settings emerged, I believe that note should be taken of the following.

My findings indicate that introducing the notion of equal partnerships, that integrate the relevant ethical principles with what you need to know about yourself in order to recognise and understand yourself and the research community you are involved in, contributes to the way in which international development and research borders in education can be transcended and partnership synergy be operationalised. Self-reflexive researchers in education who reflect not only upon their own subjectivities and how these affect the educational research projects they are involved in, but also on their connection to the cultural-historical contexts within which they work, therefore play a major role in bringing about sustainable educational change in complex social settings. Being culturally competent, developing positive interpersonal connections, and conceptualising and collaboratively facilitating methodologically sound research and monitoring and evaluation strategies within which the relevant power differentials are recognised and cultural assumptions are made, contribute to the dynamic creation of the synergy needed for effective and sustainable international collaborative partnerships in education.

\section{Acknowledgements}

I would like to express my deep gratitude to every colleague who was involved in this project. It was an exceptionally positive learning journey made possible by my British as well as my Palestinian partners in the project. I also would like to thank colleagues who served as critical readers of this reflective article, which was developed and finalised more than a year after the completion of the original project. The financial support received from the World Bank and the Palestinian Government (WBTF 097315), and the administrative support of Canterbury Christ Church University in the United Kingdom for the original project, as briefly described in this article, and North-West University, South Africa, in finalising this manuscript is acknowledged.

\section{References}

Anderson, L. (2006). Analytic autoethnography. Journal of Contemporary Ethnography, 35(4), 373-395.

Beaver, D. (2004). Does collaborative research have greater epistemic authority? Scientometrics, 60(3), 399-408.

Chan, S. (2002). On different types of international relations scholarship. Journal of Peace Research, 39(6), 747-756.

Engelbrecht, P. (2007). Creating collaborative partnerships in inclusive schools. In P. Engelbrecht \& L. Green (Eds.), Responding to the challenges of inclusive education in Southern Africa (pp. 175-183). Pretoria, South Africa: Van Schaik.

Engelbrecht, P., Wilson, V., \& Mahon, T. (2014). Strengthening school-based practice of pre-service teacher education in Palestine. Unpublished final evaluation project report for the Palestinian Ministry of Education and Higher Education and the World Bank. Canterbury, UK: Canterbury Christ Church University.

Glasbergen, P., Biermann, F., \& Mol, A. P. J. (Eds.). (2007). Partnerships, governance and sustainable development: Reflections on theory and practice. Cheltenham, UK: Edward Elgar.

Heron, J., \& Reason, P. (2006). The practice of co-operative inquiry: Research 'with' rather than 'on' people. In P. Reason \& H. Bradbury (Eds.), Handbook of action research (pp. 144-154). London, UK: Sage. 
Hodgson, N., \& Standish, P. (2006). Induction into educational research networks: The striated and the smooth. Journal of Philosophy of Education, 40(4), 563-574.

Jeffrey, P. (2003). Smoothing the waters: Observations on the process of cross-disciplinary research collaboration. Social Studies of Science, 33(4), 539-562.

Kanan, H. M., \& Baker, A. M. (2002). Palestinian novice teachers' perception of a good mentor. Journal of Education for Teaching: International Research andPpedagogy, 28(1), 35-43.

Lasker, R. D., Weiss, E. S., \& Miller, R. (2001). Partnership synergy: A practical framework for studying and strengthening the collaborative advantage. The Milbank Quarterly, 79(2), 179-205.

Merriam, S. B. (2009). Qualitative research: A guide to design and implementation. San Francisco, USA: Jossey-Bass.

Mertens, D. M. (2009). Transformative research and evaluation. New York, USA: Guilford.

Richards, R. J. (2012). 'You look very well for a transplant': Auto-ethnographic narrative \& identity in chronic kidney disease, kidney failure \& life post-transplant (Unpublished doctoral dissertation). Stellenbosch University, South Africa.

Rigby, J., \& Edler, J. (2005). Peering inside research networks: Some observations on the effect of the intensity of collaboration on the variability of research quality. Research Policy, 34(6), 784-794.

Robinson-Pant, A., \& Singal, N. (2013). Research ethics in comparative and international education: Reflections from anthropology and health. Compare: A Journal of Comparative and International Education, 43(4), 443-463.

Srivastava, M., De Boer, A., \& Pijl, S. J. (2015). Inclusive education in developing countries: A closer look at its implementation in the last 10 years. Educational Review, 67(2), 179-195.

Symonette, H. (2004). Walking pathways toward becoming a culturally competent evaluator: Boundaries, borderlands, and border crossings. In M. Thompson-Robinson, R. Hopson, \& S. SenGupta (Eds.), In search of cultural competence in evaluation (pp. 95-111). San Francisco, USA: Wiley.

Tomaselli, K. G. (2015). Hacking through academentia: Autoethnography, data and social change. Educational Research for Social Change, 4(2), 61-74. 